\section{(6) OPEN ACCESS}

\title{
Topical diquafosol for patients with obstructive meibomian gland dysfunction
}

\author{
Reiko Arita, ${ }^{1,2,3}$ Jun Suehiro, ${ }^{4}$ Tsuyoshi Haraguchi, ${ }^{4}$ Shuji Maeda, ${ }^{5}$ Koshi Maeda, $^{5}$ \\ Hideaki Tokoro, ${ }^{4}$ Shiro Amano ${ }^{2}$
}

${ }^{1}$ Department of Ophthalmology, Itoh Clinic, Saitama, Japan 2Department of Ophthalmology, University of Tokyo School of Medicine, Tokyo, Japan

${ }^{3}$ Department of Ophthalmology, Keio University, Tokyo, Japan

${ }^{4}$ Eye care company, Topcon Corporation Tokyo, Japan

${ }^{5}$ Maeda Ophthalmic Clinic, Fukushima, Japan

\section{Correspondence to} Dr Reiko Arita, Department of Ophthalmology, Itoh Clinic, 626-11 Minaminakano, Minuma-ku, Saitama city, Saitama 337-0042, Japan; ritoh@za2.so-net.ne.jp

Received 28 September 2012 Revised 7 February 2013 Accepted 17 March 2013 Published Online First 12 April 2013

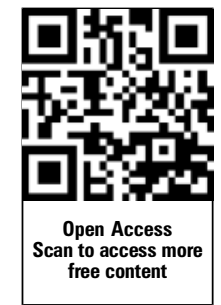

To cite: Arita R, Suehiro J, Haraguchi $\mathrm{T}$, et al. $\mathrm{Br} J$ Ophthalmol 2013;97: 725-729.

\section{ABSTRACT}

Aims To evaluate the effect of topical diquafosol in patients with meibomian gland dysfunction (MGD) using tear film parameters and quantitatively analyse the meibomian gland morphology.

Subjects and Methods The subjects were 19 eyes of 10 patients diagnosed with obstructive MGD. All subjects were given $3 \%$ diquafosol ophthalmic solution with instructions to use one drop four times a day. Ocular symptoms were scored from 0 to 14 . Lid margin abnormalities were scored from 0 to 4 . Changes in the meibomian glands were scored using non-contact meibography (meiboscore). Superficial punctate keratopathy (SPK) was scored from 0 to 3. Meibum was graded from 0 to 3. Tear film production was evaluated by Schirmer's test. Quantitative image analysis of the meibomian glands was performed using the original software.

Results 10 patients completed more than 4 months of therapy. Ocular symptoms, lid margin abnormalities, SPK score and meibum grade were decreased. Break-up time and tear film meniscus were increased. Mean ratio of the meibomian gland area was significantly increased after treatment $(p<0.0001)$.

Conclusions Quantitative image analysis was useful for evaluating the morphological changes of the meibomian glands. Topical diquafosol therapy was effective for patients with obstructive MGD.

\section{INTRODUCTION}

Meibomian glands are important structures that secrete lipid into the tear film, which prevents excessive evaporation of the tear film by forming a thin oily layer on the tear film. ${ }^{1}$ Obstructive meibomian gland dysfunction (MGD) is characterised by the condensation of meibomian lipids, resulting in the hyposecretion of lipid in the tears. ${ }^{2}$ Obstructive MGD is a major cause of lipid layer deficiency and evaporative dry eye, ${ }^{3-6}$ and may result in unstable tear film, damage to the ocular surface epithelium, chronic blepharitis, contact lens intolerance and giant papillary conjunctivitis. ${ }^{7-12}$

Conventional therapy for MGD includes warm compresses, lid hygiene, artificial tear eye drops and systemic tetracycline and doxycycline. Such therapeutic measures, however, are often unsatisfactory or poorly tolerated. ${ }^{13}$ Topical azithromycin, a macrolide antibiotic with presumed anti-inflammatory effects, was very recently reported to be effective for MGD. ${ }^{14}{ }^{15}$ Other studies have reported the use of topical cyclosporine for MGD. ${ }^{16} 17$

Diquafosol, which is commercially available in Japan as a novel dry eye therapy, is a $\mathrm{P}_{2} \mathrm{Y}_{2}$ purinergic receptor agonist that activates $\mathrm{P}_{2} \mathrm{Y}_{2}$ receptors on the ocular surface, ${ }^{18}{ }^{19}$ leading to rehydration through activation of the fluid pump of the accessory lacrimal glands on the conjunctival tissue and conjunctival goblet cell secretion of ocular mucins. The ability of the $\mathrm{P}_{2} \mathrm{Y}_{2}$ receptor activation to increase ocular lipid production (the outermost layer of the tear film) is less well established, but animal experiments have revealed the presence of $\mathrm{P}_{2} \mathrm{Y}_{2}$ receptors in the meibomian glands. ${ }^{20}$

The present study was designed to evaluate the effect of diquafosol 3\% ophthalmic solution (Diquas, Santen, Osaka, Japan) when applied to the eye and lid margin for the treatment of MGD for more than 4 months. Here, we evaluated the symptoms and clinical signs of MGD, including a quantitative morphological analysis of the meibomian glands using a non-invasive meibography system and original image analysis software.

\section{METHODS}

\section{Subjects}

The study analysed 19 eyes of 10 patients ( 3 men and 7 women; mean \pm SD of age, $72.7 \pm 4.8$ years) diagnosed with obstructive MGD. The diagnosis of MGD was based on the presence of ocular symptoms, lid margin abnormalities (irregular lid margin, vascular engorgement, plugged meibomian gland orifices and anterior or posterior replacement of the mucocutaneous junction), poor meibum expression and the loss of meibomian glands based on noninvasive meibography. ${ }^{21}$ Exclusion criteria included ocular allergies, contact lens wear, continuous use of eye drops, history of eye surgery and systemic or ocular diseases that might interfere with tear film production or function. Patients with excessive meibomian lipid secretion were also excluded.

Written informed consent was obtained from all subjects before examination. This study was approved by the institutional review board of the University of Tokyo and adhered to the tenets of the Declaration of Helsinki.

\section{Treatment protocol}

All subjects received 3\% diquafosol ophthalmic solution (Diquas; Santen Pharmaceuticals, Osaka, Japan) with instructions to use one drop four times a day. Diquas eye drops contains $0.002 \%$ benzalkonium chloride as a preservative.

\section{Examinations}

Examinations were performed sequentially as follows. All patients were questioned regarding the presence of 14 ocular symptoms: ocular fatigue, discharge, foreign body sensation, dryness, uncomfortable sensation, sticky sensation, pain, epiphora, 
itching, redness, heavy sensation, glare, too much blinking and history of chalazion or hordeolum. Symptoms were scored from 0 through 14 according to the number of these symptoms present, as described before. ${ }^{22}$

Four lid margin abnormalities (irregular lid margin, vascular engorgement, plugging of meibomian gland orifices and anterior or posterior replacement of the mucocutaneous junction) were scored from 0 through 4 according to the number of these abnormalities present in each eye. Superficial punctate keratopathy (SPK) in the cornea was scored from 0 to 3. The break-up time (BUT) of the tear film was measured three times consecutively after the instillation of fluorescein, and the mean value was used for analysis.

The upper and lower eyelids were everted, and the meibomian glands were observed using a non-contact meibography system (BG-4M, TOPCON, Tokyo, Japan). Partial or complete meibomian gland loss was scored using the following grades (meiboscore) for each eyelid as previously described: grade 0 (no meibomian gland loss), grade 1 (area of meibomian gland loss less than $1 / 3$ of the total meibomian gland area), grade 2 (area of meibomian gland loss between $1 / 3$ and $2 / 3$ of the total area) and grade 3 (area of meibomian gland loss over $2 / 3$ of the total area). ${ }^{22}$ Meiboscores for the upper and lower eyelids were summed to obtain a score from 0 through 6 for each eye. Tear film production was evaluated by Schirmer's test.

Digital pressure was applied to the upper tarsus, and the degree of ease in expressing meibomian secretion (meibum) was evaluated semiquantitatively as follows ${ }^{23}$ : grade 0 , clear meibum easily expressed; grade 1, cloudy meibum expressed with mild pressure; grade 2, cloudy meibum expressed with more than moderate pressure; and grade 3, no meibum expression, even with hard pressure. All examinations were completed on the same day, usually within $10 \mathrm{~min}$.

\section{Quantitative evaluation of meibomian gland area}

The border of the total analysis area was manually determined. Each separate meibomian gland within the total analysis area was also manually determined (figure 1). After the binarised meibomian glands were separated as independent closed curves, our original software labelled each meibomian gland and calculated the area of the closed meibomian glands. The ratio of the meibomian gland area relative to the total analysis area was then calculated.

\section{Statistical analysis}

Mean ratios of the meibomian gland area relative to the total analysis area were compared between pre-treatment and posttreatment using a paired t test. Mean increases in the ratio of meibomian gland area relative to the total analysis area were compared between the upper and lower eyelids using a paired $\mathrm{t}$ test. A p value of $<0.05$ was considered significant. Data are shown as mean $\pm \mathrm{SD}$ unless otherwise specified.

\section{RESULTS}

Ten patients completed more than 4 months of therapy (4-16 months). Patient characteristics and changes in tear film parameters are shown in table 1 .

\section{Changes in tear film parameters following treatment}

In 19 eyes of 10 patients, ocular symptoms, lid margin abnormalities, SPK score and meibum grade were decreased (table 1 and figure 2). BUT and tear film meniscus were increased. Schirmer's test value did not change. In 13 eyes of 9 patients, the meiboscore was decreased.

\section{Quantitative evaluation of meibomian gland area}

Quantitative analysis indicated that the meibomian gland areas were increased in all the 19 eyelids (upper/lower) of 10 patients (figures 3 and 4). The mean ratio of the meibomian gland area relative to the total analysis area in all 19 eyelids was 36.9 $\pm 10.1 \%$ before treatment and $41.5 \pm 9.2 \%$ after treatment $(\mathrm{P}<0.00000001)$. The mean increase in the ratio of the meibomian gland area relative to the total analysis area in 19 eyelids was $4.6 \pm 4.0 \%$ (range $0.1 \%-15.2 \%$ ). The mean increase in the ratio of meibomian gland area relative to the total analysis area in the upper and lower eyelids was $2.6 \pm 1.7 \%$ and $6.7 \pm 4.6 \%$, respectively $(\mathrm{p}=0.0000026)$.
Figure 1 Meibomian gland area measurement process. (A) Original meibography image. (B) Border for the analysis was manually determined. (C) Separate meibomian glands within the total analysis area were manually detected. (D) After the binarised meibomian glands were separated as independent closed curves, our original software labelled each meibomian gland and calculated the area of the closed meibomian glands. The ratio of the meibomian gland area relative to the total analysis area was then calculated.
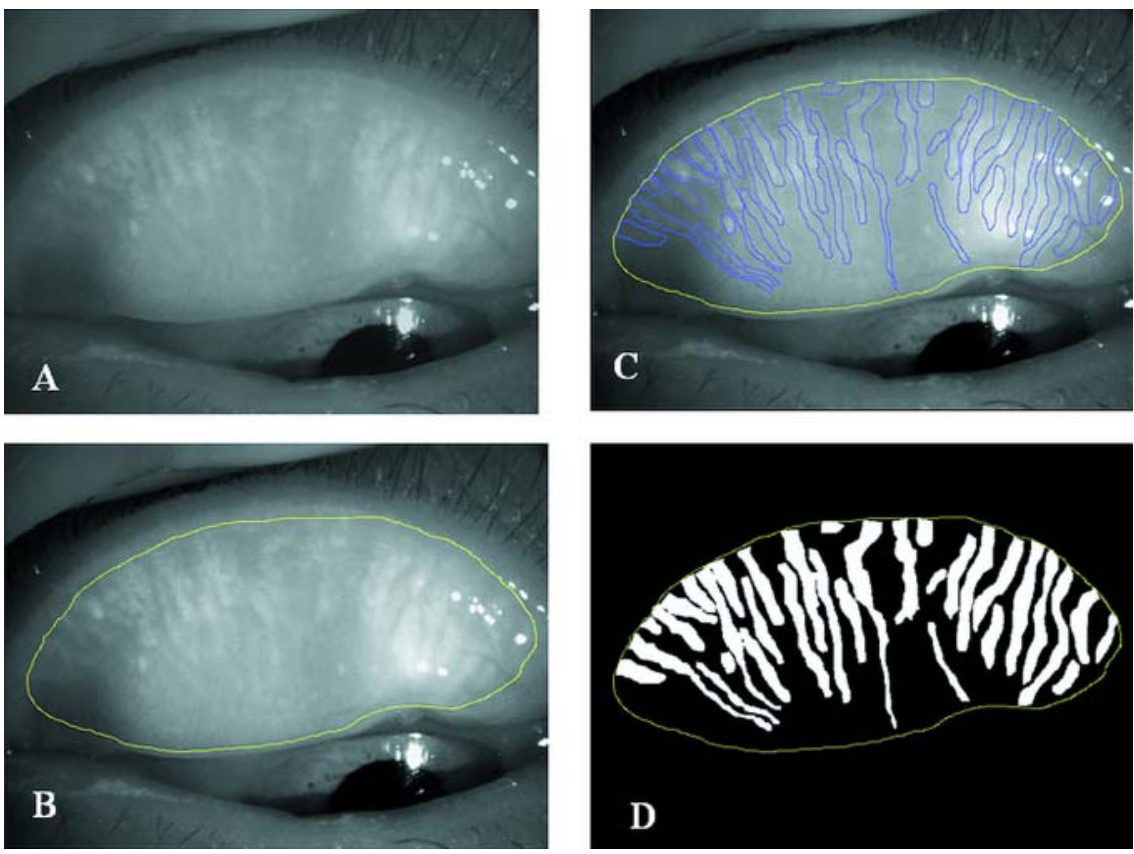
Table 1 Demographical data and changes in tear film parameters

\begin{tabular}{|c|c|c|c|c|c|c|c|c|c|c|c|c|c|c|c|c|c|c|c|c|}
\hline & \multirow[b]{2}{*}{ Age } & \multirow[b]{2}{*}{ Sex } & \multirow[b]{2}{*}{ Eye } & \multirow[b]{2}{*}{ FU (weeks) } & \multicolumn{2}{|c|}{ Symptoms } & \multicolumn{2}{|c|}{$\begin{array}{l}\text { Lid margin } \\
\text { abnormalities }\end{array}$} & \multicolumn{2}{|c|}{ BUT (s) } & \multicolumn{2}{|l|}{ SPK } & \multicolumn{2}{|c|}{$\begin{array}{l}\text { Meniscus } \\
(\mathrm{mm})\end{array}$} & \multicolumn{2}{|c|}{$\begin{array}{l}\text { Schirmer's } \\
\text { test }(\mathrm{mm})\end{array}$} & \multicolumn{2}{|c|}{$\begin{array}{l}\text { Meibum } \\
\text { grade }\end{array}$} & \multicolumn{2}{|c|}{ Meiboscore } \\
\hline & & & & & Pre & Post & Pre & Post & Pre & Post & Pre & Post & Pre & Post & Pre & Post & Pre & Post & Pre & Post \\
\hline \multirow[t]{2}{*}{1} & 80 & $\mathrm{~F}$ & $\mathrm{R}$ & 32 & 5 & 0 & 3 & 1 & 3 & 6 & 1 & 0 & 0.3 & 0.3 & 8 & 8 & 2 & 0 & 5 & 4 \\
\hline & & & $\mathrm{L}$ & 32 & 4 & 1 & 1 & 1 & 3 & 6 & 0 & 0 & 0.2 & 0.3 & 7 & 7 & 2 & 0 & 5 & 4 \\
\hline \multirow[t]{2}{*}{2} & 73 & M & $\mathrm{R}$ & 60 & 6 & 0 & 4 & 1 & 2 & 7 & 1 & 0 & 0.1 & 0.3 & 8 & 5 & 2 & 0 & 5 & 4 \\
\hline & & & $\mathrm{L}$ & 60 & 6 & 0 & 4 & 1 & 2 & 6 & 1 & 0 & 0.1 & 0.2 & 6 & 8 & 2 & 1 & 4 & 4 \\
\hline \multirow[t]{2}{*}{3} & 73 & M & $\mathrm{R}$ & 36 & 6 & 1 & 2 & 1 & 2 & 7 & 1 & 0 & 0.1 & 0.3 & 10 & 10 & 1 & 0 & 3 & 2 \\
\hline & & & $\mathrm{L}$ & 36 & 6 & 1 & 2 & 1 & 3 & 7 & 0 & 0 & 0.1 & 0.2 & 12 & 11 & 1 & 0 & 2 & 2 \\
\hline \multirow[t]{2}{*}{4} & 71 & $F$ & $\mathrm{R}$ & 64 & 5 & 1 & 2 & 1 & 3 & 6 & 1 & 0 & 0.1 & 0.2 & 10 & 10 & 2 & 1 & 2 & 2 \\
\hline & & & $\mathrm{L}$ & 64 & 4 & 1 & 3 & 1 & 3 & 5 & 0 & 0 & 0.1 & 0.2 & 6 & 10 & 2 & 0 & 4 & 4 \\
\hline \multirow[t]{2}{*}{5} & 66 & $F$ & $\mathrm{R}$ & 64 & 5 & 0 & 3 & 1 & 1 & 5 & 1 & 0 & 0.1 & 0.3 & 5 & 5 & 2 & 0 & 5 & 4 \\
\hline & & & L & 64 & 5 & 0 & 2 & 1 & 2 & 6 & 0 & 0 & 0.1 & 0.3 & 8 & 8 & 2 & 1 & 5 & 4 \\
\hline \multirow[t]{2}{*}{6} & 72 & $F$ & $\mathrm{R}$ & 20 & 5 & 1 & 3 & 1 & 3 & 7 & 2 & 0 & 0.1 & 0.3 & 12 & 12 & 1 & 0 & 2 & 2 \\
\hline & & & $\mathrm{L}$ & 20 & 5 & 0 & 3 & 1 & 3 & 8 & 2 & 0 & 0.1 & 0.3 & 12 & 12 & 1 & 0 & 2 & 1 \\
\hline 7 & 68 & $F$ & $\mathrm{~L}$ & 24 & 4 & 1 & 3 & 2 & 3 & 5 & 2 & 1 & 0.1 & 0.2 & 5 & 5 & 2 & 1 & 5 & 4 \\
\hline \multirow[t]{2}{*}{8} & 81 & $M$ & $\mathrm{R}$ & 24 & 6 & 1 & 2 & 1 & 3 & 7 & 0 & 0 & 0.1 & 0.3 & 8 & 10 & 2 & 1 & 4 & 4 \\
\hline & & & $\mathrm{L}$ & 24 & 6 & 1 & 2 & 1 & 3 & 7 & 1 & 0 & 0.1 & 0.2 & 16 & 15 & 2 & 1 & 4 & 3 \\
\hline \multirow[t]{2}{*}{9} & 74 & $F$ & $\mathrm{R}$ & 28 & 5 & 0 & 3 & 0 & 2 & 7 & 1 & 0 & 0.1 & 0.2 & 6 & 6 & 2 & 1 & 4 & 3 \\
\hline & & & L & 28 & 4 & 0 & 2 & 0 & 2 & 7 & 1 & 0 & 0.1 & 0.2 & 6 & 8 & 1 & 0 & 4 & 3 \\
\hline \multirow[t]{2}{*}{10} & 68 & $\mathrm{~F}$ & $\mathrm{R}$ & 28 & 4 & 1 & 2 & 1 & 3 & 7 & 2 & 0 & 0.1 & 0.3 & 9 & 9 & 1 & 0 & 2 & 1 \\
\hline & & & $\mathrm{L}$ & 28 & 5 & 1 & 2 & 1 & 3 & 7 & 2 & 1 & 0.1 & 0.2 & 10 & 10 & 1 & 0 & 3 & 2 \\
\hline
\end{tabular}

Symptoms (0-14), lid margin abnormalities (0-4), $\mathrm{m}$ score (0-3), meiboscore (0-6).

BUT, break-up time; F, female; FU, follow-up duration; L, left eye; M, male; R, right eye; SPK, superficial punctate keratopathy (0-3). 
Figure 2 Representative case of a 66-year-old woman with obstructive meibomian gland dysfunction (case \#5). (A) Lid margin vascularity and pluggings of the orifices were observed before therapy. (B) Tear meniscus height was very low $(0.1 \mathrm{~mm})$. (C) Lid margin vascularity and pluggings of the orifices decreased after 8 months of therapy. (D) Tear meniscus height was increased $(0.3 \mathrm{~mm})$.
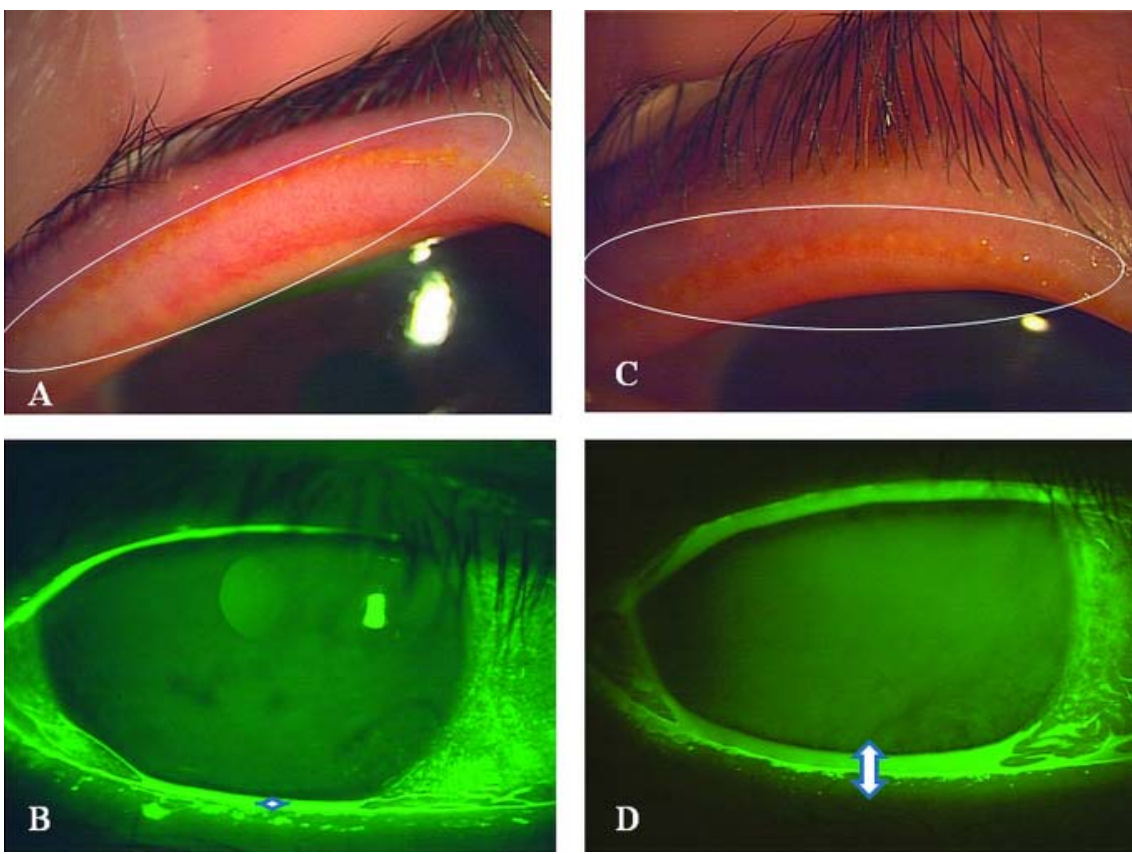

\section{DISCUSSION}

Diquafosol therapy for more than 4 months was effective for the patients with obstructive MGD. The subjective symptoms and objective findings were improved after treatment. Diquafosol is a $\mathrm{P}_{2} \mathrm{Y}_{2}$ purinergic receptor agonist that activates $\mathrm{P}_{2} \mathrm{Y}_{2}$ receptors on the ocular surface, including the meibomian glands, to increase lipid production. These findings suggest that topical diquafosol activated some existing meibomian glands to produce meibomian lipid.

We evaluated the morphology of the meibomian glands using our originally developed quantitative image analysis software.

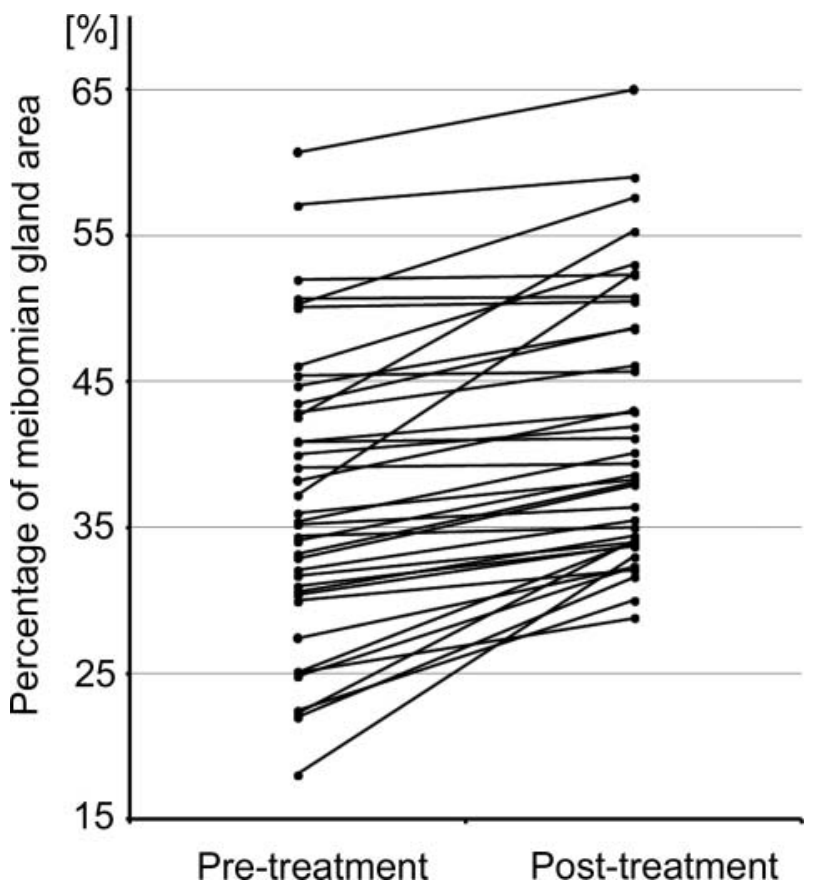

Figure 3 Changes in meibomian gland area before and after Diquas treatment. Changes in meibomian gland area were significantly increased after the Diquas treatment in the upper and lower eyelids.
This method was more objective and reproducible than the meiboscore. On the other hand, the objectivity and reproducibility of meibum scoring are low because the expression pressure on the eyelid is not defined. Moreover, meibum score is determined by the meibum expression solely at the centre of eyelids, although meibomian gland changes are diffuse in MGD. The originally developed quantitative analysis software was able to analyse the upper and lower eyelids from the nasal side to the temporal side. Moreover, we previously examined the repeatability of the image analysis using the software in five eyes of five patients with MGD, and the intraexaminer coefficients of variation for the quantitative analysis of the upper and lower meibomian gland area was 0.28 and $0.51 \%$, respectively, indicating high repeatability of the quantitative analysis of the meibomian gland area. In the present study, the mean increase in the ratio of the meibomian gland area relative to the total analysis area in 19 eyelids was 4.6\%, which is noteworthy given the high repeatability of the quantitative analysis method used in this current study.

The mean increase in the ratio of the meibomian gland area relative to the total analysis area in the lower eyelid was significantly greater than that in the upper eyelid, likely due to the fact that the eye drops remain longer in the lower eyelid than in the upper eyelid.

Diquafosol activates $\mathrm{P}_{2} \mathrm{Y}_{2}$ receptors on the ocular surface, leading to rehydration via activation of the fluid pump of the accessory lacrimal glands on the conjunctival tissue and conjunctival goblet cell secretion of ocular mucins. These mechanisms are thought to increase the BUT and the tear meniscus, and decrease SPK within a month in the patients of the present study. In contrast, Schirmer's values have not been changed. A previous study showed that diquafosol did not change the Schirmer's value in the normal rats and dry eye model rats. ${ }^{24}$ Moreover, another previous study showed that diquafosol increased tear volume in rats deprived of lacrimal glands. ${ }^{25}$ These results suggest that diquafosol increases tear volume not by increasing the secretion from the lacrimal glands but by increasing the fluid secretion via the stimulation of net ion transport across the conjunctival cells. Because it is assumed that Schirmer's strip stimulates tear secretion mainly 
Figure 4 Quantitative image analysis of the meibomian glands before and after therapy with topical diquafosol in case \#2. (A and B) Meibomian gland area was $22.0 \%$ before therapy. ( $C$ and $D$ ) Meibomian gland area had increased to $31.6 \%$ after 4 months of therapy with topical diquafosol.
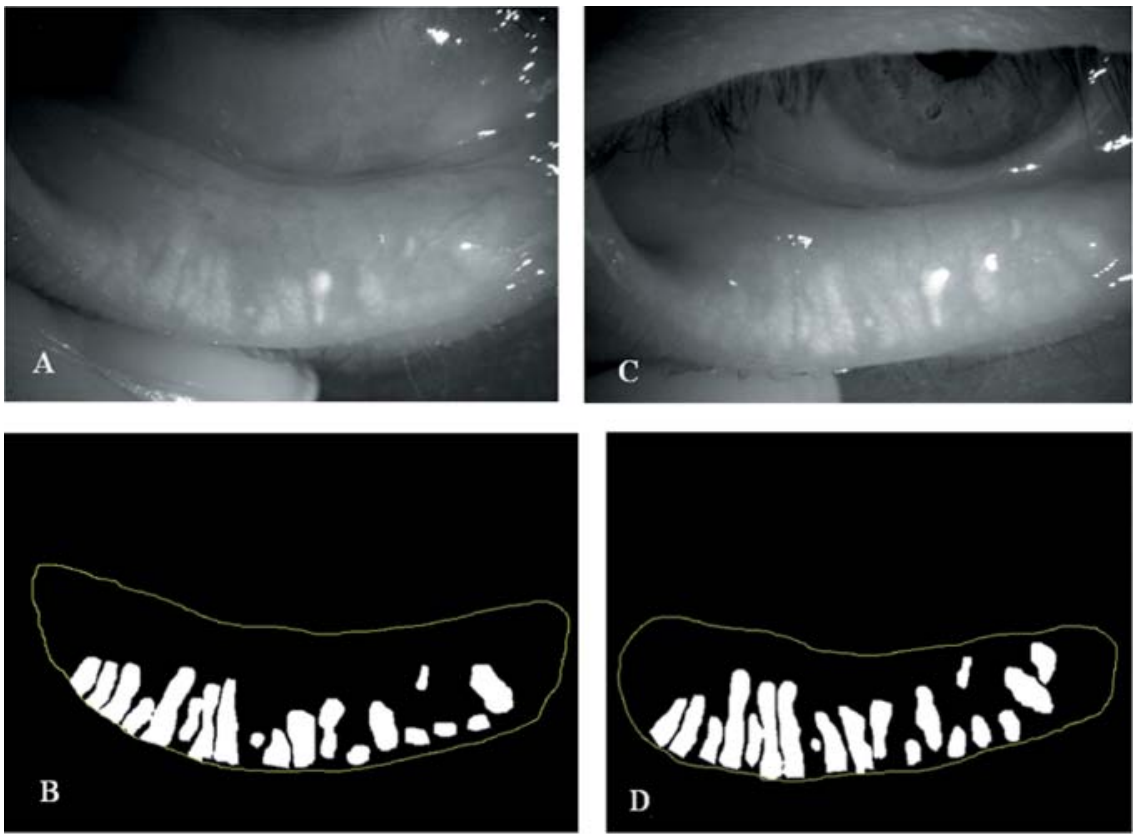

from lacrimal gland, it seems reasonable that Schirmer's value have not changed after the treatment with diquafosol in this study. Several months, however, were required to activate and reproduce meibomian lipids and to change the morphology of the meibomian glands, perhaps because the alteration of lipid composition followed by morphological changes of meibomian glands takes much longer time than the rehydration of fluid and mucins.

There are a couple of limitations in this study. One is that this study had no control group treated with the conventional MGD treatment such as warm compress and lid hygiene. The other limitation is the small number of the subjects. Further studies are necessary to fully investigate the effect of topical diquafosol on the ocular surface and in the meibomian glands of patients with MGD.

In conclusion, quantitative image analysis was useful to evaluate the morphological changes in the meibomian glands. Topical diquafosol therapy for more than 4 months was effective for patients with obstructive MGD.

Contributors Conception and design, acqusition of data (AR), analysis and interpretation of data (AR, AS, SJ, HT, TH, MS, MK). Drafting the article (AR), revising the article (AS, SJ, HT, TH, MS, MK). Final approval of the version (AR, AS, SJ, HT, TH, MS, MK

\section{Competing interests None.}

Patient consent Obtained.

Ethics approval University of Tokyo.

Provenance and peer review Not commissioned; externally peer reviewed.

Open Access This is an Open Access article distributed in accordance with the Creative Commons Attribution Non Commercial (CC BY-NC 3.0) license, which permits others to distribute, remix, adapt, build upon this work non-commercially, and license their derivative works on different terms, provided the original work is properly cited and the use is non-commercial. See: http://creativecommons.org/ licenses/by-nc/3.0/

\section{REFERENCES}

1 Mishima S, Maurice DM. The oily layer of the tear film and evaporation from the corneal surface. Exp Eye Res 1961;1:39-45.

2 McCulley JP. Meibomitis. In: Kaufman HE, Barron BA, McDonald MB, Waltman SR, eds. The Cornea. New York, NY: Churchill Livingstone Inc., 1988:125-38.

3 Lemp MA. Report of the National Eye Institute/Industry Workshop on Clinical Trials in Dry Eyes. CLAO J 1995;21:221-32.

4 Mathers WD. Ocular evaporation in meibomian gland dysfunction and dry eye. Ophthalmology 1993;100:347-51.
5 Shimazaki J, Sakata M, Tsubota K. Ocular surface changes and discomfort in patients with meibomian gland dysfunction. Arch Ophthalmol 1995;113:1266-70.

6 Lee SH, Tseng SCG. Rose Bengal staining and cytologic characteristics associated with lipid tear deficiency. Am J Ophthalmol 1997;124:736-50.

7 Mathers WD, Shields WJ, Sachdev MS. Meibomian gland dysfunction in chronic blepharitis. Cornea 1991;10:277-85.

8 Mathers WD, Billborough M. Meibomian gland function and giant papillary conjunctivitis. Am J Ophthalmol 1992;114:188-92.

9 Korb DR, Henriquez AS. Meibomian gland dysfunction and contact lens intolerance. J Am Optom Assoc 1980;51:243-51.

10 Henriquez AS, Korb DR. Meibomian glands and contact lens wear. Br J Ophthalmol 1981;65:108-11.

11 Zengin N, Tol H, Gunduz K, et al. Meibomian gland dysfunction and tear film abnormalities in rosacea. Cornea 1995:14:144-6.

12 Ong BL. Relation between contact lens wear and meibomian gland dysfunction. Optom Vis Sci 1996;73:208-10.

13 Paranjpe DR, Foulks GN. Therapy for meibomian gland disease. Ophthalmol Clin North Am 2003;16:37-42.

14 Foulks GN, Borchman D, Yappert M, et al. Topical azithromycin and oral doxycycline therapy of meibomian gland dysfunction: a comparative clinical and spectroscopic pilot study. Cornea 2013:32:44-53.

15 Foulks GN, Borchman D, Yappert M, et al. Topical azithromycin therapy for meibomian gland dysfunction: clinical response and lipid alterations. Cornea 2010;29:781-8

16 Rubin M, Rao SN. Efficacy of topical cyclosporin $0.05 \%$ in the treatment of posterior blepharitis. J Ocul Pharmacol Ther 2006;22:47-53.

17 Perry HD, Doshi-Carnevale S, Donnenfeld ED, et al. Efficacy of commercially available topical cyclosporine A $0.05 \%$ in the treatment of meibomian gland dysfunction. Cornea 2006;25:171-5

18 Kamiya K, Nakanishi M, Ishii R, et al. Clinical evaluation of the additive effect of diquafosol tetrasodium on sodiumhyaluronate monotherapy in patients with dry eye syndrome: a prospective, randomized, multicenter study. Eye (Lond) 2012;26:1363-8.

19 Nakamura M, Imanaka T, Sakamoto A. Diquafosol ophthalmic solution for dry eye treatment. Adv Ther 2012:29:579-89.

20 Cowlen MS, Zhang VZ, Warnock L, et al. Localization of ocular P2Y2 receptor gene expression by in situ hybridization. Exp Eye Res 2003;77:77-84

21 Arita R, Itoh K, Maeda S, et al. Proposed diagnostic criteria for obstructive meibomian gland dysfunction. Ophthalmology 2009;116:2058-63.

22 Arita R, Itoh K, Inoue K, et al. Noncontact infrared meibography to document age-related changes of the meibomian glands in a normal population. Ophthalmology 2008;115:911-15.

23 Shimazaki J, Goto E, Ono M, et al. Meibomian gland dysfunction in patients with Sjogren syndrome. Ophthalmology 1998;105:1485-8.

24 Fujihara T, Murakami T, Fujita $\mathrm{H}$, et al. Improvement of corneal barrier function by the P2Y(2) agonist INS365 in a rat dry eye model. Invest Ophthalmol Vis Sci 2001:42:96-100

25 Li Y, Kuang K, Yerxa B, et al. Rabbit conjunctival epithelium transports fluid, and P2Y2(2) receptor agonists stimulate $\mathrm{Cl}(-)$ and fluid secretion. Am J Physiol Cell Physiol 2001;281:C595-602. 